\title{
Robotic manipulation planning for shaping deformable linear objects with environmental contacts
}

\author{
Jihong Zhu, Benjamin Navarro, Robin Passama, Philippe Fraisse, André Crosnier and Andrea Cherubini
}

\begin{abstract}
Humans use contacts in the environment to modify the shape of deformable objects. Yet, few papers have studied the use of contacts in robotic manipulation. In this paper, we investigate the problem of robotic manipulation of cables with environmental contacts. Instead of avoiding contacts, we propose a framework that allows the robot to use them for shaping the cable. We introduce an index to quantify the contact mobility of a cable with a circular contact. Based on this index, we present a planner to plan robot motions. The planner is aided by a visionbased contact detector. The framework is validated with robot experiments on different desired cable configurations.
\end{abstract}

Index Terms-Dexterous Manipulation, Contact Modeling, Manipulation Planning

\section{INTRODUCTION}

Humans are able to manipulate objects with high dexterity. For decades, we have been trying to give robots similar capabilities. Robotic manipulation has been studied intensively with the focus on rigid objects. However, humans encounter all kinds of deformable objects and manipulate them on a regular basis. Therefore, to fully embed robots with humanlike manipulation skills, we should pay considerable attention to deformable objects manipulation. The fields of application include areas like surgical operation, agriculture, food making, household services and industrial automation.

One of the biggest challenges in deformable objects manipulation is the infinite degree of freedoms in the object deformation versus finite inputs from robot manipulators/hands. Often, when dealing with deformable objects, humans not only apply both hands, but also use contacts in the environment to regulate the object shape. For instance, when folding a towel, we place it on a flat surface to constrain its deformation due to gravity.

Deformable objects with linear shapes such as cables, wires, etc., are often referred to as deformable linear objects (DLOs) in the literature. Yet, for simplicity, we will denote them as cables in this paper. In cable harness, cables need to follow a designated path defined by a set of contacts (see Fig. 1). Our

Manuscript received: Jun, 7, 2019; Revised Aug, 22, 2019; Accepted Sept, 12 , 2019. This paper was recommended for publication by Editor Jingshan Li upon evaluation of the Associate Editor and Reviewers' comments. This work was supported by European Union Horizon 2020 research and innovation programme as part of the project VERSATILE under grant agreement No 731330 .

All authors are with the Laboratory for Computer Science, Microelectronics and Robotics LIRMM - Université de Montpellier CNRS, 161 Rue Ada, 34090 Montpellier, France. firstname.lastnamed lirmm.fr

Digital Object Identifier (DOI): see top of this page. objective is to establish a robotic manipulation framework that automates the cable routing process.

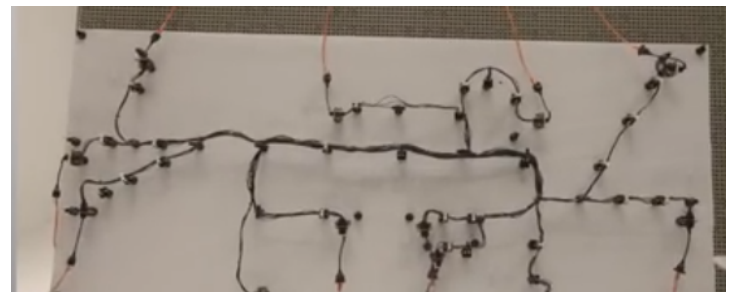

Fig. 1. Cable harness board with contacts.

We simplify this problem by considering circular contacts on the board. The robot plans its motion according to the contacts placement, detects the occurrence of a contact, modifies its manipulation behaviour accordingly and finally achieves a desired configuration of the cable.

\section{A. Related work}

We outline works that have been done in robotic cable manipulation in three sub-categories: cable modelling, manipulation planning and shape control.

The model of a cable can be either geometrical or topological. The geometrical model usually requires optimizing the system total energy. Wakamatsu et al. developed a cable model considering bend, twist and extensional deformation [1]. Later, this model was explored for grasping and manipulation planning in [2]. Yoshida et al. simulated the deformation of an elastic band using the finite element method [3]. Topological models are another option. One of the first models was developed in [4], to solve the knotting problem. The topological model is often based on knot theory [5], and the problem to solve is usually tying [6] or untying knots [7].

In manipulation planning, contact with the environment is often considered as undesired - and thus to be avoided. A collision free path planner was developed in [8] using a randomized algorithm. A planner in [9] computed a path in the shape space from one minimal energy curve to another while satisfying environmental constraints. Bretl and McCarthy showed that the shape space of an elastic rod is a six-dimensional smooth manifold [10]. Later, the authors of [11] took a step forward and proved the path-connectedness of this space.

In shape control, we distinguish model-based and modelfree methods. Nakagaki et al. used a deformation model for 
insertion tasks [12]. Navarro-Alarcon et al. developed a modelfree Fourier-based shape servoing strategy [13]. We extended that work to the shape servoing of cables in [14].

Few papers have investigated cable manipulation with contacts in the environment. In a pioneer work [15], the authors presented the specification of contact states for linear objects with polyhedral obstacles, and identified unstable/stable contact states. Acker and Henrich further proposed visual features for the detection of contact state transitions in cable manipulation [16]. In a more recent research [17], the authors planned cable manipulation strategies with a simulator in both free and contact space. Some works in deformable object manipulation exploited contact for manipulation: plastic material shaping [18] and towel folding [19] where some or all manipulation tasks were done with contact compensating the gravity. Yet, these papers do not study the specific use of contact.

\section{B. Our contribution}

To the best of authors' knowledge, contact-based cable routing relying on robotic manipulation is still an open research problem. Yet, it is widely present in the industry (e.g., in cable harness). We address this practical problem by a mathematical analysis on the contact mobility. We propose an index to quantify the mobility. This index motivates our choice of motion primitives. A motion planning framework using the primitives is designed for the robot to shape a cable by contacts. The performance of the framework is analyzed using the proposed contact mobility index.

\section{Paper organization and notation}

The paper is organized as follow. Sec. II states the contactbased cable manipulation problem and presents an overview of the framework. Sec. III defines the angular contact mobility index. Then we analyze two cable motions and their effect on the index. Sec. IV proposes motion primitives for the robot. Sec. V presents the planner which uses the motion primitives for planning robot motions. Sec. VII explains the setup and shows experiment results ${ }^{1}$. In the last section, we conclude and propose some future research directions.

We employ the following mathematical notation throughout the paper. Vectors are denoted with small bold letters, e.g. $\boldsymbol{v}$, $\boldsymbol{w}$. Matrices are with capital bold letters e.g. $\boldsymbol{A}, \boldsymbol{B}$. The vector difference is denoted by $\rightarrow$, e.g. $\vec{v} \boldsymbol{w}=\boldsymbol{w}-\boldsymbol{v}$. The Euclidean norm of a vector is given by $\|\cdot\|$, e.g. $\|\boldsymbol{v}\|,\|\overrightarrow{\boldsymbol{v}} \boldsymbol{w}\|$.

\section{PROBlem STATEMENT AND FRAMEWORK OVERVIEW}

\section{A. Problem statement}

The overall problem is depicted in Fig. 2. We consider contacts as small circles on the 2D plane. For a cable with sufficient length, given a starting end of the cable, with a list of ordered small ${ }^{2}$ circular contacts placed on the plane, the cable should be manipulated such that it follows the path defined by the contacts' position and sequence. The order of the contacts

\footnotetext{
${ }^{1}$ The video of the experiments can be found at: https://youtu.be/7CdNQ4R_ wT0

${ }^{2}$ The radius of the contact is much smaller than the length of the cable.
}

is known a priori by the robot. The cable must be shaped by each contact sequentially and in the end reach the target position.

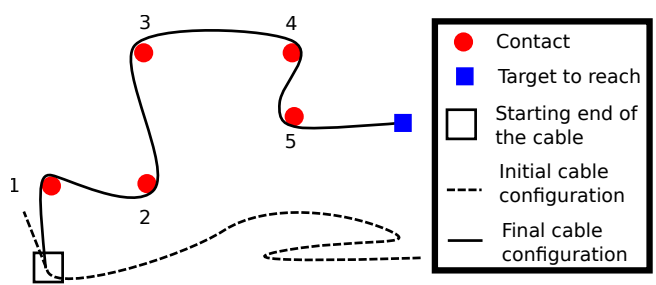

Fig. 2. A contact-based manipulation example to illustrate the problem. The order of the contacts is given by the number besides each contact. This information is provided a priori to the robot.

The cable should touch all contacts in the given order, without creating any loop. We do not consider friction in this work, and neglect the cable's deformation along the tangential direction.

\section{B. Framework overview}

We utilize a dual arm robot for the cable manipulation task. The robot is equipped with two end-effectors $\mathcal{M}$ and $\mathcal{F}$. We place $\mathcal{F}$ at a fixed pose to hold the starting end of the cable. $\mathcal{M}$ holds the other end of the cable and is free to move on the 2D manipulation plane. An in-hand camera is mounted on $\mathcal{M}$ to provide visual feedback.

The framework consists of a planner and a vision-based contact detector. The planner plans the motion for $\mathcal{M}$ given contacts and a target location. The detector identifies the occurrence of a contact.

\section{ANGULAR CONTACT MOBILITY INDEX}

One way of analyzing the contact is by its mobility index, which measures the free motion of the object in contact [20]. In this section, we introduce a novel contact mobility index based on the angular range of motion. We term it Angular Contact Mobility Index (ACMI). The robot manipulates the cable on a plane. The cable is soft and can adapt to the curvature of the contact object. In Fig. 3 we show an example. We construct a local Cartesian coordinate frame $O x y$ at the center of the object. We consider the motion of the cable relative to the contact. The direction of motion is a unit vector $\boldsymbol{v}(\phi)=[\cos \phi \sin \phi]^{T}$ with $\phi(0 \leq \phi<2 \pi)$ the angle between the vector and $x$ axis. For any point $\rho$ on the contact curve, the direction of the contact force is denoted $\boldsymbol{n}(\boldsymbol{\rho})$.

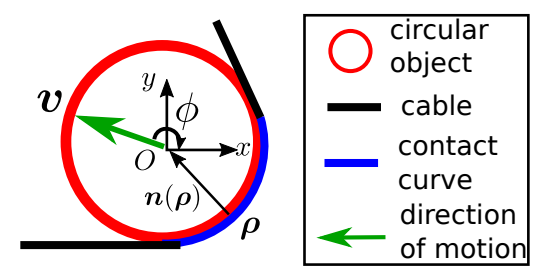

Fig. 3. Example of a circular object in contact with a cable. The green vector is a candidate direction of relative object/cable motion. Many others directions are possible. 
Definition 1. Angular Contact Mobility Index (ACMI): the angle (in radians) that represents the range of directions the object can move to break free from the contact.

In Fig. 4, we consider - in four cases - the directions of motion of the contact object (red circle) with regards to the cable. The direction $\boldsymbol{v}$ is parameterized by $\phi$. We derive the ACMI for these cases and finally provide a general expression of the ACMI.

When there is no contact (Fig. 4a), the break free (from contact) directions are given by the following set:

$$
\mathcal{R}=\{\phi \in[0,2 \pi)\} .
$$

Since the object can move in all directions, the ACMI equals $2 \pi$.

For a single contact point $\rho$ (Fig. 4b), the set of break free directions is defined by:

$$
\mathcal{R}(\boldsymbol{\rho})=\{\phi \in[0,2 \pi): \boldsymbol{n}(\boldsymbol{\rho}) \cdot \boldsymbol{v}(\phi)>0\} .
$$

This defines an open set of directions with only positive components in the direction of $\boldsymbol{n}$. The angular range in the $\mathcal{R}(\boldsymbol{\rho})$ is $\pi$. The ACMI equals $\pi$.

For the curved contact in Fig. 4c, the set of break free directions is expressed by:

$$
\mathcal{R}=\bigcap_{\rho=\rho_{1}}^{\rho_{2}} \mathcal{R}(\rho) .
$$

Noting $\psi$ the contact curve angular range, if $\psi \in(0, \pi]$, then the ACMI is $\pi-\psi$.

Instead, if $\psi \in(\pi, 2 \pi]$ (see Fig. 4d), the ACMI equals 0 . No matter which direction the object moves, it cannot break free from the contact.

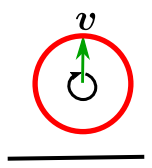

(a) No contact

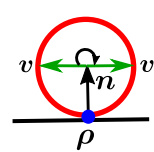

(b) Point contact
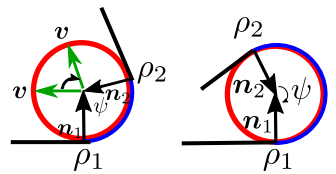

$\begin{array}{ll}\text { (c) } 0<\psi \leq \pi & \text { (d) } \pi<\psi \leq 2 \pi\end{array}$
Fig. 4. The ACMI in four contact cases, (a): no contact; (b): point contact; (c): curved contact with $0<\psi \leq \pi$; (d): curved contact with $\pi<\psi \leq 2 \pi$.

To sum up, we provide a quantitative definition of the ACMI:

$$
\text { ACMI }= \begin{cases}2 \pi, & \text { No contact } \\ \pi, & \text { Point contact, } \\ \max (0, \pi-\psi) & \text { Curved contact. }\end{cases}
$$

Using the ACMI, we quantify the free motion range due to the contact. The higher the index, the larger the range of motion sufficient to break free from contact (i.e., it is easier to lose contact).

Below we analyze two motions of the cable and their effects.

The first motion is termed rotation. Starting from an initial contact point $\left(\rho_{2}\right)$ scenario depicted in Fig. 5, the ACMI is $\pi$. Consider the left segment of the cable fixed, and perform an anti-clockwise rotation with center at the contact for the right segment: we expect the contact point to become a curve. Then the length of the curve grows due to this movement, so the ACMI decreases. The reverse motion will make the contact curve shrink, and the ACMI increase.

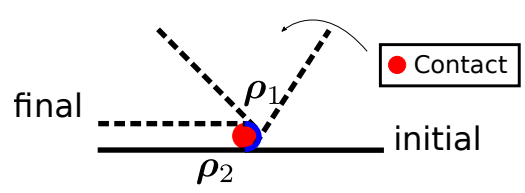

Fig. 5. The effect of rotation on the ACMI.

The second motion is termed sliding. In case of contact point (Fig. 6a), sliding corresponds to:

$$
\mathcal{R}(\boldsymbol{\rho})=\{\phi \in[0,2 \pi): \boldsymbol{n}(\boldsymbol{\rho}) \cdot \boldsymbol{v}(\phi)=0\} .
$$

For a curved contact with two end points (Fig. 6b), we denote two edges of the curve as $\boldsymbol{\rho}_{1}$ and $\boldsymbol{\rho}_{2}$ with $\boldsymbol{n}_{1}$ and $\boldsymbol{n}_{2}$ being the directions of the associated contact force. Sliding corresponds to the direction within:

$$
\mathcal{R}_{1} \bigcup \mathcal{R}_{2}
$$

with

$$
\begin{aligned}
& \mathcal{R}_{1}=\left\{\phi \in[0,2 \pi): \boldsymbol{n}_{1} \cdot \boldsymbol{v}(\phi)=0\right\}, \\
& \mathcal{R}_{2}=\left\{\phi \in[0,2 \pi): \boldsymbol{n}_{2} \cdot \boldsymbol{v}(\phi)=0\right\} .
\end{aligned}
$$

In both figures we show an example of contact after sliding in a specific direction. The sliding motion maintains the contact curve (point) and the ACMI stays unchanged.

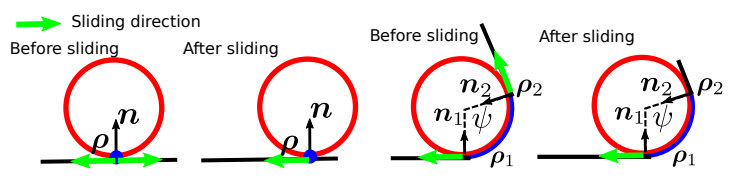

(a) Point contact case

(b) Curved contact case

Fig. 6. The effect of sliding on the ACMI.

In the next sections, we will relate robotic cable manipulation with these two motions. Using the ACMI, we show in Sec. VII-C that with the proposed framework, the robot constructs and utilizes contacts for shaping the cable to reach a desired configuration.

\section{MOTION PRIMITIVES}

In Fig. 7, we define the manipulation plane coordinate frame $\mathcal{F} x y$ with origin at the position of the fixed end-effector $\mathcal{F}$, and we attach a local coordinate frame $\mathcal{M} x y$ to the end-effector $\mathcal{M}$. The pose of $\mathcal{M}$ in $\mathcal{F} x y$ is then $\boldsymbol{q}_{\mathcal{M}}=\left[x_{\mathcal{M}} y_{\mathcal{M}} \theta_{\mathcal{M}}\right]^{T} \in$ $\mathbb{R}^{3}$, with $\theta_{\mathcal{M}}$ the counterclockwise orientation of $\mathcal{M} x y$ with regard to $\mathcal{F} x y$.

The fixed end-effector $\mathcal{F}$ is designed such that it can either hold or release one end of the cable. The moving end-effector $\mathcal{M}$ always holds the other end of the cable. When $\mathcal{F}$ is holding, the length of the cable between the two end effectors is fixed. Then $\mathcal{M}$ rotates the cable (Fig. 7a) with a fixed point on the cable as the rotation center. When $\mathcal{F}$ releases, it allows $\mathcal{M}$ to 


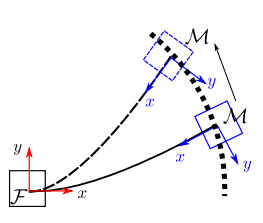

(a)

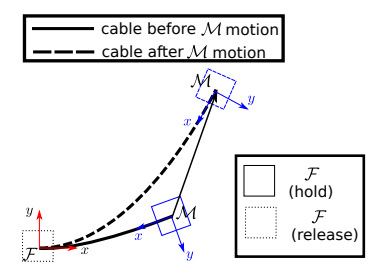

(b)
Fig. 7. Motion primitives: (a). End-effector $\mathcal{F}$ holds while end-effector $\mathcal{M}$ rotates the cable, (b). End-effector $\mathcal{F}$ releases while end-effector $\mathcal{M}$ pulls the cable.

pull the cable so that the length of the cable between the two end-effectors increases (Fig. 7b).

We define two motion primitives for the dual arm robot to accomplish the task:

- rotate: $\mathcal{F}$ hold $+\mathcal{M}$ rotate.

- pull: $\mathcal{F}$ release $+\mathcal{M}$ pull.

\section{Planner}

In this section, we introduce planners for the robot motion. For each contact, the robot executes the pull motion primitive to reach an initial pose for making contact and then the rotate motion primitive to construct and use the contact. Since the cable length is much larger than the contact radius, the radius is assumed neglectable in the section.

The flow chart describing the overall motion generated by the planner for $n$ ordered contacts is shown in Fig. 8 .

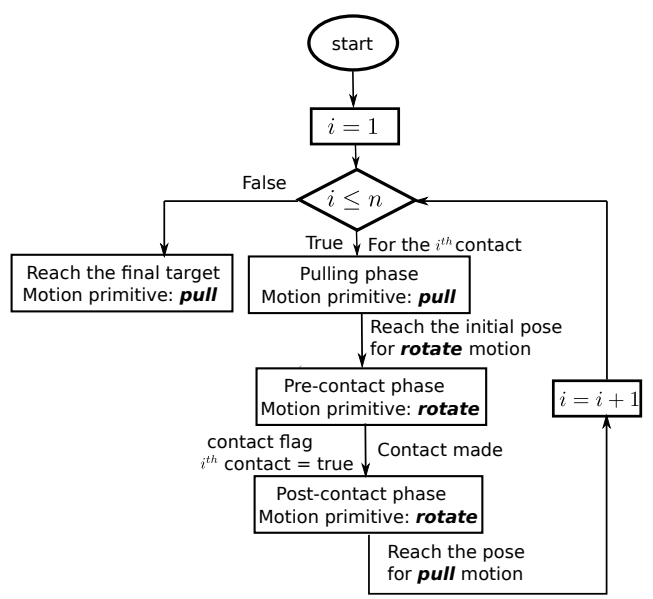

Fig. 8. Flow chart depicting the steps to reach the final target by contact-based manipulation with $n$ contacts.

\section{A. Initial pose planner}

The initial pose planner plans a discrete target pose $\boldsymbol{q}_{\mathcal{M}}$ around each contact. We denoted the planned pose as $\boldsymbol{q}^{*}=$ $\left[\begin{array}{ll}\boldsymbol{p}^{* T} & \theta^{*}\end{array}\right]^{T}$, with $\boldsymbol{p}^{*}=\left[\begin{array}{ll}x_{\boldsymbol{p}^{*}} & y_{\boldsymbol{p}^{*}}\end{array}\right]^{T}$ as position and $\theta^{*}$ as orientation.

Let us consider a general case in Fig. 9. We set the fixed point on the cable at $\boldsymbol{f}=\left[\begin{array}{ll}x_{f} & y_{f}\end{array}\right]^{T}$, the current contact at $\boldsymbol{c}=\left[\begin{array}{ll}x_{c} & y_{c}\end{array}\right]^{T}$, the next contact at $\boldsymbol{r}=\left[\begin{array}{ll}x_{r} & y_{r}\end{array}\right]^{T}$. The fixed point is either at origin of $\mathcal{F} x y$ or the previous contact location which regulates the cable. We use pull to reach each target planned pose.

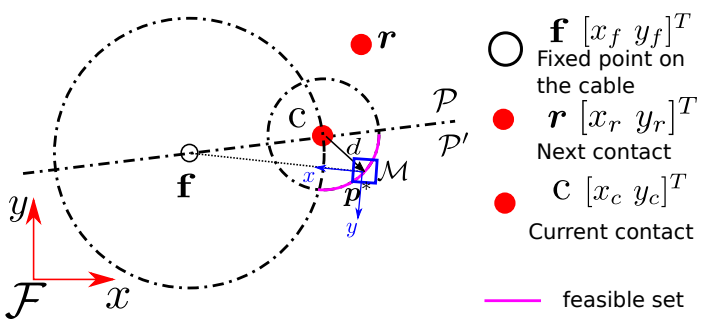

Fig. 9. Position planning for a single contact.

After having reached the planned pose we rotate with the center at $f$ to construct a contact, so the cable length is fixed. Thus, we have our first condition on $\boldsymbol{p}^{*}$ :

$$
\left\{\boldsymbol{p}^{*} \in \mathbb{R}^{2} \mid\left\|\overrightarrow{\boldsymbol{f} \boldsymbol{p}^{*}}\right\|>\|\overrightarrow{\boldsymbol{f} \boldsymbol{c}}\|\right\}
$$

Otherwise, the cable cannot reach the contact by rotation.

We separate the plane into two half planes, by a line connecting the fixed point $\boldsymbol{f}$ and the contact $\boldsymbol{c}$. We denote the half plane with the next contact as $\mathcal{P}$ and the other one as $\mathcal{P}^{\prime}$. To reach the next contact, we need:

$$
p^{*} \in \mathcal{P}^{\prime}
$$

We envision $\mathcal{M}$ to be near the contact, because if it is far, more effort is needed to bring the cable in contact. To this end, we impose a third condition:

$$
\left\{\boldsymbol{p}^{*} \in \mathbb{R}^{2} \mid\left\|\overrightarrow{c p^{*}}\right\|=d\right\}
$$

where $\|\overrightarrow{c r}\|>d>0$. In practical implementations, $d$ should be set considering the end-effector size (if too small, the endeffector may collide with the contact), and the camera field of view (large $d$ may jeopardize contact visibility).

By combining (7) - (9), we obtain a feasible set for $\boldsymbol{p}^{*}$ :

$$
\left\{\boldsymbol{p}^{*} \in \mathbb{R}^{2} \mid \mathcal{P}^{\prime} \cap\left\{\left\|\overrightarrow{\boldsymbol{f} \boldsymbol{p}^{*}}\right\|>\|\overrightarrow{\boldsymbol{f} \boldsymbol{c}}\|\right\} \cap\left\{\left\|\overrightarrow{\boldsymbol{c p}^{*}}\right\|=d\right\}\right\},
$$

which is illustrated in Fig. 9 (purple arc).

The local coordinate frame $\mathcal{M} x y$ should have its positive $x$ direction towards $f$ (the center of rotation). Thus, the target orientation $\theta^{*}$ can be calculated as $\theta^{*}=\arctan \left(y_{\boldsymbol{\Delta}} / x_{\boldsymbol{\Delta}}\right)$, with $\boldsymbol{\Delta}=\boldsymbol{f}-\boldsymbol{p}^{*}$.

Extending the method to multi-contact cases is straightforward. For each contact, the fixed point $f$ is at the previous contact (or at the origin of $\mathcal{F} x y$ for the first contact). For the last contact, the next contact $\boldsymbol{r}$ is at the target position. We calculate the planned pose for each contact in the given order. Figure 10 shows a planning example.

Once the planned pose is reached, we go to the pre-contact phase. 


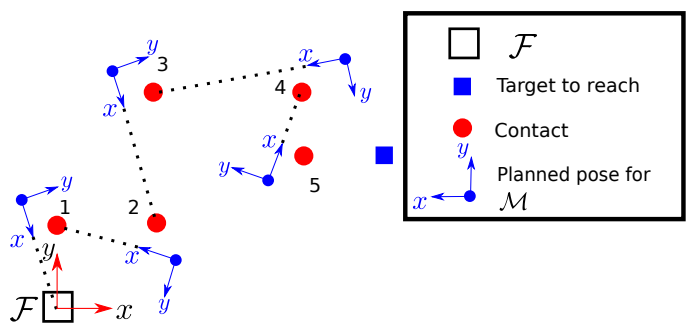

Fig. 10. Multiple contacts planning example. The order of the contacts is presented by the numbering besides each contact, and known by the robot, which can then compute the initial pose.

\section{B. Real-time local planner}

In the pre-contact phase, the local planner generates motions to reach the contact. This planner is termed real-time because it continuously receives contact detection information from a vision-based contact detector. When a contact occurs, the robot moves to the post-contact phase and the planner changes its behaviour.

1) Pre-contact phase: In this phase, we rotate the cable to construct a contact. $\mathcal{F}$ will hold the cable so that the cable length is fixed. We denote a set of planned rotational positions as:

$$
\boldsymbol{P}=\left[\begin{array}{lll}
\boldsymbol{p}^{1} & \boldsymbol{p}^{2} & \cdots
\end{array}\right]
$$

where $\boldsymbol{p}^{i}=\left[\begin{array}{ll}x_{p}^{i} & y_{p}^{i}\end{array}\right]^{T} \in \mathbb{R}^{2}$ is the $i^{t h}$ planned position on the manipulation plane. The rotational direction can be either clockwise or anti-clockwise. In Fig. 11, with initial position of $\mathcal{M}$ at $\boldsymbol{p}^{*}$, we define the two vectors $\boldsymbol{l}_{1}=\boldsymbol{p}^{*}-\boldsymbol{f} \in \mathbb{R}^{2}$ and $\boldsymbol{l}_{2}=\boldsymbol{c}-\boldsymbol{f} \in \mathbb{R}^{2}$. We construct a matrix $\boldsymbol{L}=\left[\boldsymbol{l}_{1}, \boldsymbol{l}_{2}\right] \in$ $\mathbb{R}^{2 \times 2}$. The direction is calculated by $s=\operatorname{sgn}(\operatorname{det}|\boldsymbol{L}|)$ with positive clockwise and negative anti-clockwise. The radius for the rotation is $r=\left\|\boldsymbol{p}^{*}-\boldsymbol{f}\right\|$. We set the rotational step to be $\delta \theta$. For each $\boldsymbol{p}^{i}$ we set orientation $\theta_{i}=\theta^{*}+i \delta \theta s$. The position vector $\boldsymbol{p}^{i}=\left[\begin{array}{ll}x_{p}^{i} & y_{p}^{i}\end{array}\right]^{T}$ is then

$$
\boldsymbol{p}^{i}=\boldsymbol{f}+r\left[\begin{array}{c}
\cos \theta_{i} \\
-\sin \theta_{i}
\end{array}\right] \in \mathbb{R}^{2} .
$$

The full pose vector for $\mathcal{M}$ is $\left[\boldsymbol{p}^{i^{T}} \theta_{i}\right]^{T}$. A planning example is shown on Fig. 11. $\mathcal{M}$ continues to rotate until contact occurs.

2) Contact detection: Since the contact is not on the robot but on the cable, contact forces cannot be directly measured on the robot. In addition, the contact force can be very small, which makes it hard to detect. Therefore, we use vision for the detection. We present the contact detector in Sec. VI.

3) Post-contact phase: Once a contact is made, the robot uses the contact to shape the cable, until a good configuration is obtained for reaching the next planned initial pose (or to reach the target). When a contact occurs, the robot has to adapt its manipulation behaviour. Let us denote the position of $\mathcal{M}$ at the time of contact by $\boldsymbol{p}^{\prime}$. In the post-contact phase, contact $\boldsymbol{c}=\left[\begin{array}{ll}x_{c} y_{c}\end{array}\right]^{T}$ becomes the new fixed point, thus the new center for rotation is $\boldsymbol{c}$, and the new rotation radius is $r^{\prime}=\left\|\boldsymbol{p}^{\prime}-\boldsymbol{c}\right\|$. We re-plan the robot motion with the new center $c$ and radius $r^{\prime}$ in the same manner as in the pre-contact phase, and keep the rotational direction. The example overall motion is shown in Fig. 11. As discussed in Sec. III, this post-contact rotation enlarges the contact curve, so the ACMI decreases. This way, the robot utilizes the contact for shaping the cable, and the contact is strengthened by the motion.

From the initial planner we get the position of the next planned pose $t$. End effector $\mathcal{M}$ continues to rotate until it lies on the line connecting $c$ and $t$. Then, the robot pulls the cable towards $t$. As depicted in Fig. 12, since we consider the radius of the circular contact neglectable $(r \ll l)$, then:

$$
\overrightarrow{c e}=\overrightarrow{c \rho}+\overrightarrow{\rho e} \approx \overrightarrow{\rho e} .
$$

The direction of the pull is the same as the sliding one, which we analyzed in Sec. III. Since the ACMI stays unchanged during the pull, the cable maintains contact.

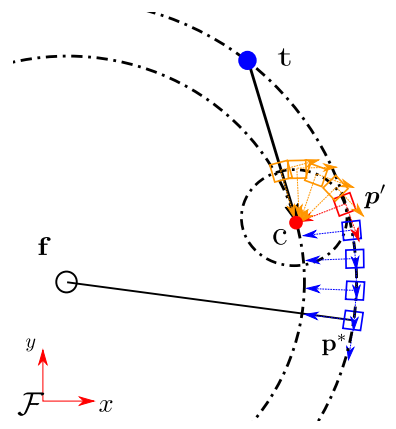

$$
\begin{aligned}
& \mathbf{f}\left[\begin{array}{ll}
x_{f} & y_{f}
\end{array}\right]^{T} \\
& \text { Fixed point on the cable } \\
& \mathbf{t}\left[\begin{array}{ll}
x_{t} & y_{t}
\end{array}\right]^{T} \\
& \text { Next planned position } \\
& \quad \mathrm{c}\left[\begin{array}{ll}
x_{c} & y_{c}
\end{array}\right]^{T} \\
& \text { Contact } \\
& \mathbf{p}^{*} \quad \text { Current planned } \\
& \text { position } \\
& \quad \begin{array}{l}
\text { pre-contact } \\
\text { planning }
\end{array} \\
& \text { post-contact } \\
& \text { planning }
\end{aligned}
$$

Fig. 11. Full rotational motion planning.

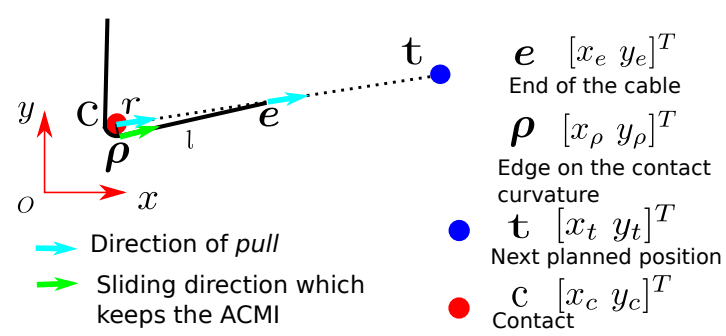

Fig. 12. The pull can be regarded as a sliding motion

\section{CONTACT DETECTOR}

We detect the contact by extracting three features on the inhand camera image, respectively: the locations of two ends of the cable segment and the contact. The detector is active only when a contact is in the image. To ease extraction, we use a white manipulation plane, a black cable and blue contacts. OpenCV [21] is used for image processing. Below is the procedure to extract these features:

- The blue contact is found via HSV (Hue, Saturation, Value) thresholding.

- The image is binarized using uniform thresholding. Then, the Canny edge detector outputs the contour(s) of the cable in the image.

- Since the relative translation between the cable end held by $\mathcal{M}$ and the camera is fixed, that cable end is selected as the bottom point on the contour in a small Region Of Interest (ROI). (Fig. 13b orange ROI). The other end of the cable is selected as the upmost point on the contour within the green ROI at the top of the image. 
The feature extraction results are shown in Fig. 13b with the end held by $\mathcal{M}$ orange, the other end green, and the contact red.

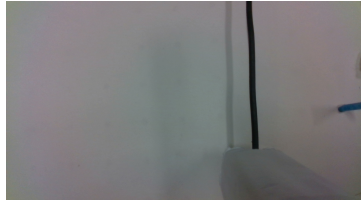

(a)

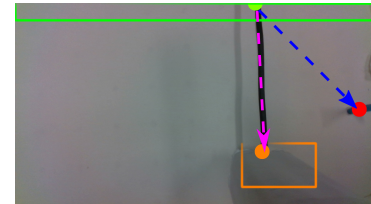

(b)
Fig. 13. Extraction of the cable ends and the contact: (a). Original image, (b). Image after processing with locations of three features and ROIs

Given the locations of the three features: orange $e_{o}=$ $\left[\begin{array}{ll}u_{o} & v_{o}\end{array}\right]^{T}$, green $\boldsymbol{e}_{g}=\left[\begin{array}{ll}u_{g} & v_{g}\end{array}\right]^{T}$, and red $\boldsymbol{e}_{r}=\left[\begin{array}{ll}u_{r} & v_{r}\end{array}\right]^{T}$ on the image, we detect a contact when the angle between $\overrightarrow{\boldsymbol{e}_{g} \boldsymbol{e}_{o}}$ (Pink dashed arrow in Fig. 13b) and $\overrightarrow{\boldsymbol{e}_{g} \boldsymbol{e}_{r}}$ (Blue dashed arrow in Fig. 13b) is smaller than a threshold (set to $0.1 \mathrm{rad}$ in the robotic experiments).

\section{ROBOT EXPERIMENTS}

\section{A. Hardware setup}

We use our BAZAR robot [22] which is equipped with two lightweight KUKA LWR IV. Planning is carried out in the task space, then projected in the joint space via inverse kinematics. To avoid kinematic singularities, we use adaptive damped least squares [23]. For a starting pose $\boldsymbol{p}_{s}$ and final desired pose $\boldsymbol{p}_{f}$. We specified the starting/final velocity and acceleration to be 0 , and the maximum (operational space) velocity $\left(0.2 \mathrm{~m} \cdot \mathrm{s}^{-1}\right)$ and acceleration $\left(0.1 \mathrm{~m} \cdot \mathrm{s}^{-2}\right)$. The trajectory generation is based on second order polynomials. The generation is done by reflexxes motion generation library [24].

A table serves as the manipulation plane. We use cylinder screws as contacts and a board with holes for easy insertion of contacts. A white wall paper covers the board, with only the contacts standing out. The size of the manipulation plane is $0.5 \mathrm{~m} \times 0.9 \mathrm{~m}$.

Figure $14 \mathrm{a}$ shows the end-effector $\mathcal{M}$. A cable can be firmly attached at the bottom of it. An Intel Realsense D435 camera is mounted, with height from the bottom adjustable $(3-27 \mathrm{~cm})$. We only use the camera RGB images. The image resolution is $1920 \times 1080 . \mathcal{F}$ is a $3 \mathrm{D}$ printed structure with 4 springs. When pressed on the table, it holds the cable; when lifted, it lets the cable slide (Fig. 14b).

Figure 15 shows the coordinate frames. An ArUco marker [25] is placed just below $\mathcal{F}$ to serve as the origin of the manipulation plane. The manipulation plane is parallel to $X Y$ plane in the robot frame.

The transformation from any position $\left[\begin{array}{lll}X & Y & Z\end{array}\right]^{T} \in \mathcal{R} X Y Z$ to $\left[\begin{array}{ll}x & y\end{array}\right]^{T} \in \mathcal{F} x y$ is $\left[\begin{array}{ll}x & y\end{array}\right]^{T}=\left[\begin{array}{ll}X & Y\end{array}\right]^{T}-\left[\begin{array}{ll}X_{\mathcal{F}} & Y_{\mathcal{F}}\end{array}\right]^{T}$, where $\left[\begin{array}{ll}X_{\mathcal{F}} & Y_{\mathcal{F}}\end{array}\right]^{T}$ is the $X Y$ position of $\mathcal{F}$ in the robot frame.

\section{B. Contact localization}

The location of contacts in the robot frame is obtained by commanding an initial upward motion of $\mathcal{M}$ to capture an image of the manipulation plane with the ArUco marker. Using

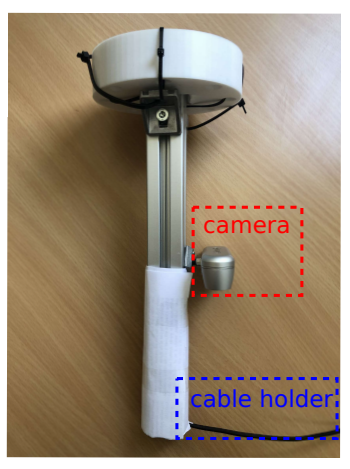

(a) End-effector $\mathcal{M}$.

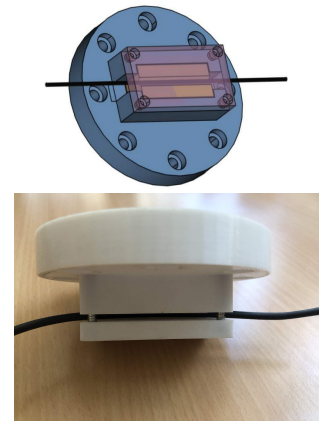

(b) End-effector $\mathcal{F}$.
Fig. 14. Designs of the two end-effectors

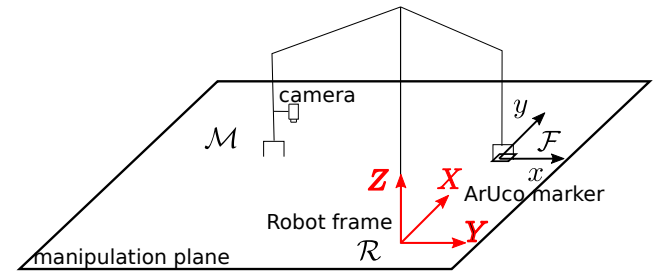

Fig. 15. Setup and coordinate frames.

this image, with simple HSV thresholding, we find the blue contact locations on the image plane. The projection equation from $3 \mathrm{D}$ to $2 \mathrm{D}$ is then:

$$
\left[\begin{array}{lll}
u & v & 1
\end{array}\right]^{T}=\boldsymbol{D} \boldsymbol{C} \boldsymbol{T}_{\mathcal{R}}^{\mathcal{C}}\left[\begin{array}{llll}
X & Y & Z & 1
\end{array}\right]^{T},
$$

where $\boldsymbol{D} \in \mathbb{R}^{3 \times 3}, \boldsymbol{C} \in \mathbb{R}^{3 \times 4}$ are respectively distortion and camera matrices, and $\boldsymbol{T}_{\mathcal{R}}^{\mathcal{C}} \in \mathbb{R}^{4 \times 4}$ is the transformation from the robot to the camera frame. Both the depth $Z$ and $\boldsymbol{T}_{\mathcal{R}}^{\mathcal{C}}$ can be obtained via ArUco. Given image coordinates $u$ and $v$, the projection equation (14) consists of two functions with two unknowns. Thus, we solve the robot frame coordinate $X$ and $Y$ from $u$ and $v$. The contacts are conventionally ordered with increasing $X$ axis coordinate.

\section{Results}

To validate our framework, we did 8 experiments with different contact configurations. Figure 16 shows the manipulation time for each experiment scenario ${ }^{3}$. The majority of time is dedicated to the rotation, during which the contact detector is active. We use a rotation step of $0.02 \mathrm{rad}$. The rotation step is chosen based on our image processing rate (on average 60 milliseconds per image) for an accurate contact detection. A late detection will put the cable in high tension. With faster image processing, we could reduce the execution time in the rotation phase by taking larger rotation steps. This could be achieved - for instance - by using smaller size images.

Figure 20 presents graphically all the multi-contact scenarios with nominal cable configurations. In the figure, the contact

\footnotetext{
${ }^{3}$ We developed a website indicating all the control and vision parameters used in our experiments: https://jihong-zhu.github.io/robotics/2019/08/17/ Experiment-contact-based-manipulation.html.
} 
and initial/target $X Y$ ( $Z$ is constant) locations are given by vectors (in meters) expressed in the robot frame $\mathcal{R} X Y Z$. The initial pose of $\mathcal{M}$ is $[-0.050 .65 \pi]^{T}$. To reach the target, we set the target orientation for $\mathcal{M}$ as $\pi$.

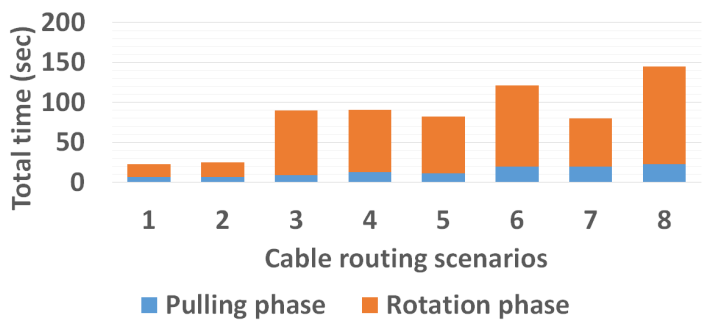

Fig. 16. Total manipulation time for each scenario. Single contact cases: 1,2. Two contacts cases: $3-5$. Three contacts cases: 6-8.

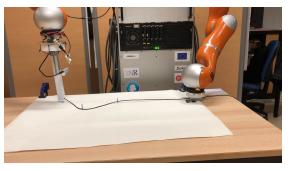

(a) Scenario 3

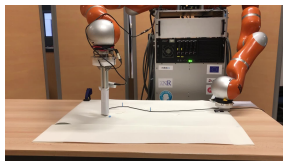

(d) Scenario 6

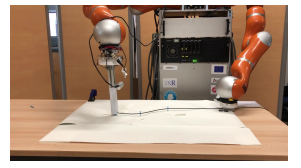

(b) Scenario 4

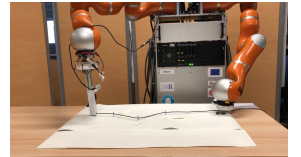

(e) Scenario 7

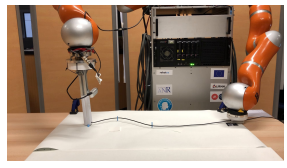

(c) Scenario 5

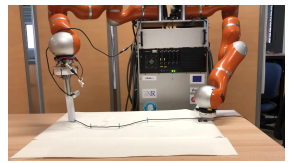

(f) Scenario 8
Fig. 17. Final cable configurations in six of the eight scenarios.

Figure 17 shows the final configuration for these scenarios. The robot achieves all these configurations by contact regulation. Figure 21 shows a step by step manipulation process for scenario 8 .

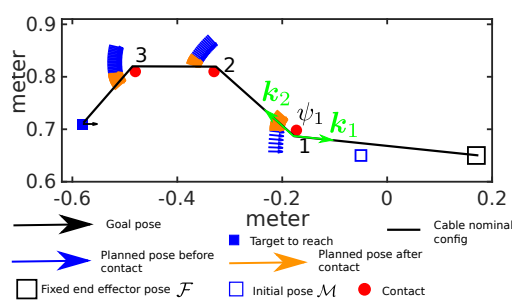

(a) Planned motion for scenario 8

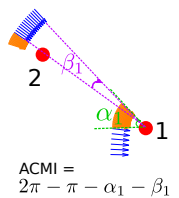
culation. (b) ACMI cal-

Fig. 18. (a): Planned motion for scenario 8; (b): Example calculation of the ACMI of contact 1 after the robot motion.

We can analyze the effect of robot motion on the ACMI for individual contact and then for the overall experiment. For an experiment with $n$ contacts, the initial overall ACMI is $2 \pi n$. We expect the ACMI to decrease as the cable is regulated by each contact. The initial overall ACMI for scenario 8 is $3 \times 2 \pi=6 \pi$. Given the motion in Fig. $18 \mathrm{a}$, we can calculate the ACMI for the $i^{\text {th }}$ contact as shown on Fig. 18b:

$$
\mathrm{ACMI}_{i}=2 \pi-\pi-\alpha_{i}-\beta_{i} .
$$

The $-\pi$ in (15) comes from the first contact detected by the vision-based detector. A point contact is then constructed.
The $\alpha_{i}>0$ is the rotation angle subsequent to the contact detection. The $\beta_{i} \in[-\pi, \pi]$ is the rotation angle after pull until the next contact is detected; $\beta_{i}$ is positive if the rotation after the pull has the same direction as before the pull, and negative otherwise. For the last contact $\beta_{n}=0 \mathrm{rad}$.

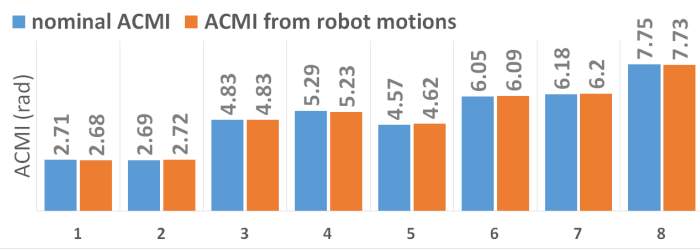

Fig. 19. The nominal ACMI and the ACMI after the manipulation.

For the example on Fig. 18b, from the motion and contact locations we are able to calculate $\alpha_{1}=0.76 \mathrm{rad}$ and $\beta_{1}=-0.0862 \mathrm{rad}$ ( $\beta$ is negative as the direction is different from the rotation direction before the pull). The ACMI for the contact is: $2 \pi-\pi-0.76+0.086=2.46 \mathrm{rad}$. ACMIs for the rest of contacts can be calculated in the same manner.

One simple measure to analyse overall contact mobility is by summing up the ACMI of each contact for a given scenario. The total ACMI for a $n$ contacts setup after robotic manipulation can be calculated by (16):

$$
\mathcal{T}=\sum_{i=1}^{n} \operatorname{ACMI}_{i}
$$

For a given topology of contact locations (order known), the start and target end-effector positions, one could calculate the nominal total ACMI by the cable configuration. Consider the example in Fig. 18, based on the nominal cable configuration (obtained by connecting the start, contacts in order and the target sequentially), the nominal ACMI for the first contact is $\psi_{1}$ which is the angle between the $\boldsymbol{k}_{1}$ and $\boldsymbol{k}_{2}$ (see Fig. 18). Similarly, using (16) we can calculate total nominal ACMI for a given the topology. Figure 19 presents a bar graph of comparison between the nominal and actual ACMI in each scenario. The difference between the nominal ACMI and ACMI calculated from the robot motion is very small. This confirms that with our framework the robot is able to construct contact and to use it for shaping the cable to reach the desired configuration.

In these experiments, we were constrained by the operational space of the robot, and by the simple mechanical structure designed to hold the cable. To extend the framework for more contacts one needs to consider: 1. re-grasping of the cable, 2. using a mobile base to enlarge the robot operational space.

\section{CONCLUSION AND FUTURE WORK}

In this paper, we address contact-based robotic manipulation of cables. Two motion primitives are selected based on a contact analysis. Then, we propose a novel planning strategy with a vision-based contact detector. Experiments are carried out to validate the approach.

The paper is one of the pioneer works which considers environmental contacts in deformable object manipulation. 


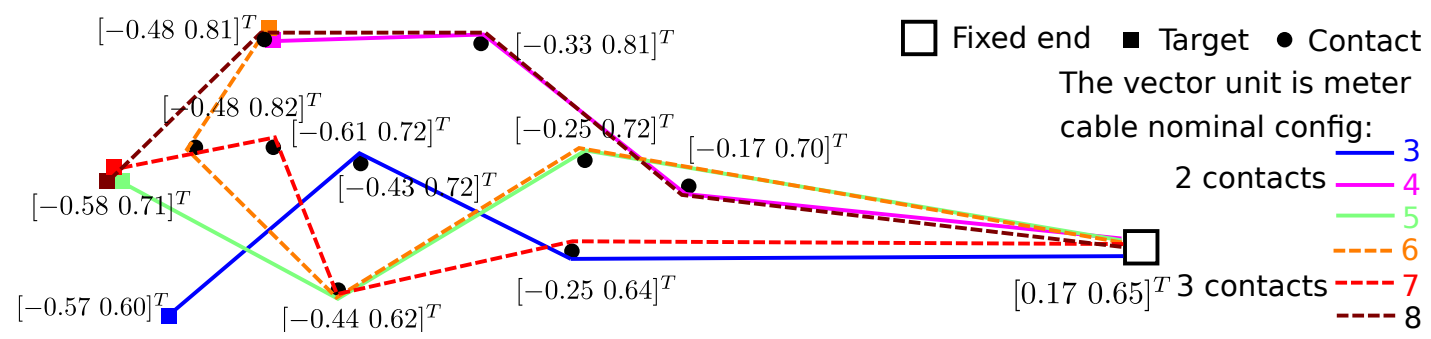

Fig. 20. Manipulation experiments with more than one contact. Contacts are denoted with black dots, and the nominal cable configuration is drawn with solid ( 2 contacts) and dashed ( 3 contacts) lines

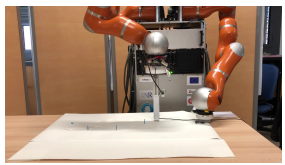

(a) Starting pose.

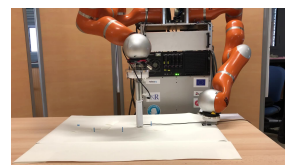

(b) First contact.

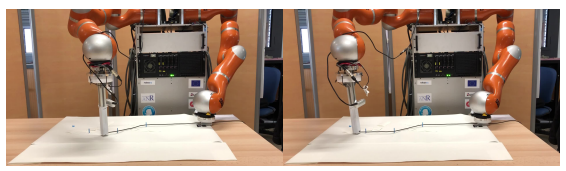

(c) Second contact.

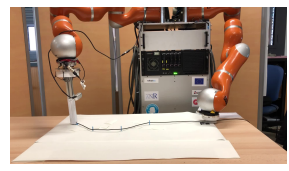

(e) Reach the final pose.

Fig. 21. Manipulation process for scenario 8 .

We believe contacts play a vital role in deformable objects manipulation. Currently, since we use a fixed base, the number of contacts is limited, as is the robot operational space. With a mobile base, one could enhance the robot operational range. In addition, since the rationale behind a human using a specific contact for shaping is probably closely related to cognitive science, some inspiration can be drawn from [26] for studying how robots should use different contacts. In a nutshell, contact for deformable objects manipulation is a rich area with a lot of new research opportunities. We hope this work can be a starting point for future research.

\section{REFERENCES}

[1] H. Wakamatsu, S. Hirai, and K. Iwata, "Modeling of linear objects considering bend, twist, and extensional deformations," in IEEE Int. Conf. on Robotics and Automation, vol. 1, 1995, pp. 433-438.

[2] H. Wakamatsu and S. Hirai, "Static modeling of linear object deformation based on differential geometry," Int. J. of Robotics Research, vol. 23, no. 3, pp. 293-311, 2004.

[3] E. Yoshida, K. Ayusawa, I. G. Ramirez-Alpizar, K. Harada, C. Duriez, and A. Kheddar, "Simulation-based optimal motion planning for deformable object," in IEEE Int. Workshop on Advanced Robotics and its Social Impacts (ARSO), 2015.

[4] J. E. Hopcroft, J. K. Kearney, and D. B. Krafft, "A case study of flexible object manipulation," Int. J. of Robotics Research, vol. 10, no. 1, pp. 41-50, 1991.

[5] K. Murasugi, Knot theory and its applications. Springer Science \& Business Media, 2007.

[6] F. Wang, E. Burdet, R. Vuillemin, and H. Bleuler, "Knot-tying with visual and force feedback for vr laparoscopic training," in 2005 IEEE Engineering in Medicine and Biology, 2006, pp. 5778-5781.

[7] H. Wakamatsu, E. Arai, and S. Hirai, "Knotting/unknotting manipulation of deformable linear objects," Int. J. of Robotics Research, vol. 25, no. 4, pp. 371-395, 2006.

[8] F. Lamiraux and L. E. Kavraki, "Planning paths for elastic objects under manipulation constraints," Int. J. of Robotics Research, vol. 20, no. 3 , pp. 188-208, 2001.

[9] M. Moll and L. E. Kavraki, "Path planning for deformable linear objects," IEEE Trans. on Robotics, vol. 22, no. 4, pp. 625-636, 2006.

[10] T. Bretl and Z. McCarthy, "Quasi-static manipulation of a kirchhoff elastic rod based on a geometric analysis of equilibrium configurations," Int. J. of Robotics Research, vol. 33, no. 1, pp. 48-68, 2014.

[11] A. Borum and T. Bretl, "The free configuration space of a kirchhoff elastic rod is path-connected," in IEEE Int. Conf. on Robotics and Automation (ICRA), 2015.
[12] H. Nakagaki, K. Kitagaki, T. Ogasawara, and H. Tsukune, "Study of deformation and insertion tasks of a flexible wire," in IEEE Int. Conf. on Robotics and Automation, vol. 3, 1997, pp. 2397-2402.

[13] D. Navarro-Alarcon and Y.-H. Liu, "Fourier-based shape servoing: A new feedback method to actively deform soft objects into desired 2-D image contours," IEEE Trans. on Robotics, 2017.

[14] J. Zhu, B. Navarro, P. Fraisse, A. Crosnier, and A. Cherubini, "Dualarm robotic manipulation of flexible cables," in IEEE/RSJ Int. Conf. on Intelligent Robots and Systems, 2018.

[15] D. Henrich, T. Ogasawara, and H. Worn, "Manipulating deformable linear objects-contact states and point contacts," in IEEE International Symposium on Assembly and Task Planning, 1999.

[16] J. Acker and D. Henrich, "Manipulating deformable linear objects: characteristic features for vision-based detection of contact state transitions," in IEEE Int. Symposium on Assembly and Task Planning, 2003.

[17] O. Roussel and M. Taïx, "Deformable linear object manipulation planning with contacts," in Robot Manipulation: What has been achieved and what remains to be done? Full day workshop at IEEE/RSJ Int. Conf. on Intelligent Robots and Systems (IROS), 2014.

[18] A. Cherubini, J. Leitner, V. Ortenzi, and P. Corke, "Towards vision-based manipulation of plastic materials," in IEEE/RSJ Int. Conf. on Intelligent Robots and Systems (IROS). IEEE, 2018.

[19] J. Maitin-Shepard, M. Cusumano-Towner, J. Lei, and P. Abbeel, "Cloth grasp point detection based on multiple-view geometric cues with application to robotic towel folding," in IEEE Int. Conf. on Robotics and Automation, 2010.

[20] E. Rimon and J. W. Burdick, "Mobility of bodies in contact. II. how forces are generated by curvature effects," IEEE Transactions on Robotics and Automation, vol. 14, no. 5, pp. 709-717, 1998.

[21] G. Bradski, "The OpenCV Library," Dr. Dobb's Journal of Software Tools, 2000

[22] A. Cherubini, R. Passama, B. Navarro, M. Sorour, A. Khelloufi, O. Mazhar, S. Tarbouriech, J. Zhu, O. Tempier, A. Crosnier et al., "A collaborative robot for the factory of the future: Bazar," Int. J. of Advanced Manufacturing Technology, pp. 1-17, 2019.

[23] S. Chiaverini, O. Egeland, and R. K. Kanestrom, "Achieving userdefined accuracy with damped least-squares inverse kinematics," in 5th International Conference Advanced Robotics, June 1991, pp. 672-677 vol.1.

[24] T. Kröger, "Opening the door to new sensor-based robot applications the reflexxes motion libraries," in IEEE Int. Conf. on Robotics and Automation (ICRA), 2011.

[25] S. Garrido-Jurado, R. M. noz Salinas, F. Madrid-Cuevas, and R. MedinaCarnicer, "Generation of fiducial marker dictionaries using mixed integer linear programming," Pattern Recognition, vol. 51, pp. 481 - 491, 2016.

[26] K. P. Tee, J. Li, L. T. P. Chen, K. W. Wan, and G. Ganesh, "Towards emergence of tool use in robots: Automatic tool recognition and use without prior tool learning," in 2018 IEEE Int. Conf. on Robotics and Automation (ICRA), pp. 6439-6446. 